\section{Neurotrophic keratitis}

S Bonini ${ }^{1,3}$, P Rama ${ }^{2}$, D Olzi ${ }^{1}$ and A Lambiase ${ }^{1,3}$

an impairment of corneal sensitivity. ${ }^{2}$ Systemic diseases such as diabetes, multiple sclerosis, and leprosy may decrease sensory nerve function or damage sensory fibres leading to corneal anaesthesia., ${ }^{3,4}$ The corneal epithelium is the first target of the disease showing dystrophic changes and defects with poor tendency to spontaneous healing. The progression of the disease may lead to corneal ulcers, melting, and perforation. ${ }^{5}$ While the clinical diagnosis is easily oriented from the history and clinical findings, the management of this condition is one of the most difficult and challenging among all corneal diseases.

\section{Pathophysiology}

The cornea is provided with the richest innervation of all body tissues (40 times more than the tooth pulp and 400 times more than skin). It is generally accepted that corneal sensory nerves play a key role in maintaining the anatomical integrity and function of the cornea and particularly of the epithelium. Several reports have shown that loss of corneal sensory innervation leads to a decrease of the vitality, metabolism, and mitosis of epithelial cells and consequently to an epithelial breakdown. ${ }^{6,7}$ The corneal epithelium thickness is decreased and epithelial cells show intracellular swelling, loss of microvilli, and an abnormal production of the basal lamina. ${ }^{8,9}$ Conjunctival changes include a decrease of goblet cell density and loss of cell-surface microplicae. ${ }^{10}$

Several studies have focused on the role of sensory neuromediators in the corneal epithelium pathophysiology. These studies have shown the depletion of substance P (SP) and acetylcholine (Ach) in the rat cornea after sensory nerve injury. ${ }^{11,12}$ In vitro, SP, cholecystokinin gene-related peptide (CGRP), and Ach induce epithelial proliferation. ${ }^{13-15}$ In contrast, in animal studies, SP treatment failed to promote corneal epithelial healing. ${ }^{16}$ Chikama et al ${ }^{17}$ showed a complete recovery of neurotrophic keratitis in one patient with a combination of SP and insulin-like growth

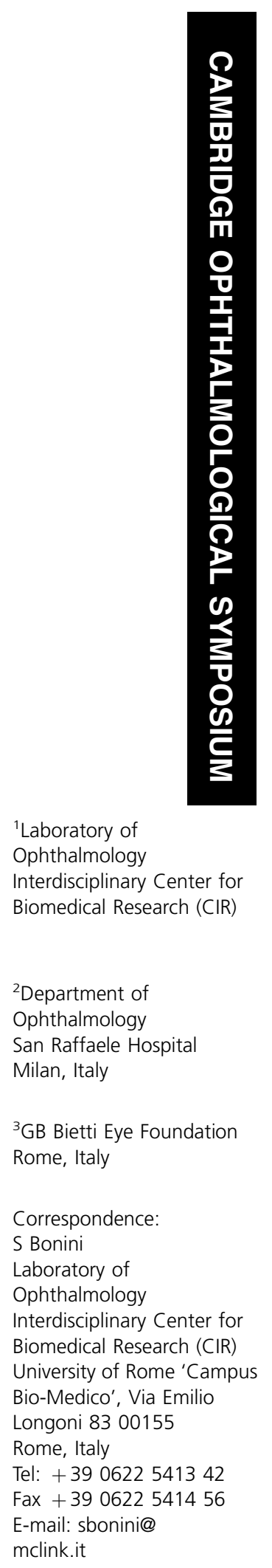

Received: 28 February 2003 Accepted in revised form: 28 February 2003 
Table 1 A etiology of corneal sensory deficits

Genetic
Riley-Day syndrome (familial dysautonomia)
Goldenhar-Gorlin syndrome
Mobius syndrome
Familial corneal hypoestesia
Systemic
Diabetes
Leprosy
Vitamin A deficiency
Central nervous system
Neoplasm
Aneurysms
Stroke
Postneurosurgical procedures
For acoustic neuroma
For trigeminal neuralgia
Other surgical injury to the trigeminal nerve
Ocular
Post-herpes infections (herpes simplex and herpes zoster)
Chemical and physical burns
Abuse of topical anaesthetics
Drug toxicity (timolol, betaxolol, diclofenac sodium,
sulphacetamide $30 \%$ )
Postsurgical or laser treatment (trauma of ciliary nerves)
Corneal incisions
Chronic ocular surface injury or inflammation
Contact lens wear
Orbital neoplasia
Corneal dystrophies (lattice, granular)

factor-1 (IGF-1) eye drops. They hypothesized that the combination of SP and IGF-1 stimulates corneal epithelial cell migration and the expression of integrin $\alpha 5$ and $\beta 1$ essential for the attachment of epithelial cells to the extracellular matrix proteins.

Experimental and clinical evidence suggests a bidirectional control of corneal epithelium proliferation: sensory neuromediators promote epithelial cell mitosis, while, on the other hand, sympathetic mediators, epinephrine and norepinephrine, reduce epithelial cell mitosis. ${ }^{13}$ In addition, in animal studies, corneal epithelium changes induced by lesion of the sensory nerves can be reduced by cervical sympathetic denervation. $^{18}$

We have recently evaluated the potential role of nerve growth factor (NGF) in the treatment of neurotrophic keratitis. ${ }^{19,20}$

NGF induces in vitro and in vivo recovery of sensory neurons and induces the production of Ach in the central nervous system and SP in the peripheral nervous system. ${ }^{21,22}$ In addition, NGF plays an important role in the balance between sensory and sympathetic innervation, by modulating their functions. ${ }^{23}$ In rodents, corneal sensory innervation is dependent on NGF action $^{24}$ and, in vitro, NGF promotes proliferation and differentiation of rabbit corneal epithelial cells. ${ }^{25}$ In our study, in 43 patients affected by neurotrophic ulcers, topical administration of NGF restored corneal integrity and improved corneal sensitivity. ${ }^{19,20}$

To summarize, corneal sensory nerve damage induces marked changes in the levels of neuromediators that lead to an impairment of epithelial cell vitality - clinically represented by the development of recurrent or persistent epithelial defects.

\section{Presentation}

The literature does not report data regarding the epidemiology of neurotrophic keratitis. We have recently followed 43 patients affected by neurotrophic keratitis with epithelial defects or corneal ulcers. ${ }^{19}$ In six of these patients $(13.9 \%)$ we did not find any justification of corneal anaesthesia and they were classified as idiopathic, all of them were female and the onset of the disease was before the age of 6 years. The other 37 patients were affected by neurotrophic keratitis as a result of: herpetic keratitis (27.0\%); following repeated corneal surgery (21.6\%); anaesthetic abuse (16.2\%); chemical burns (13.3\%); neurosurgery (10.8\%) diabetes (8.1\%); radiotherapy $(2.7 \%)$. No significant difference was observed among the sexes (17 males; 20 females).

According to the Mackie classification, it is possible to classify neurotrophic keratitis into three stages (Table 2). ${ }^{1,5}$ Stage 1 is characterized by punctate keratopathy, epithelial hyperplasia and irregularity, superficial neovascularization, and stromal scarring (Figure 1).

Stage 2 is characterized by a persistent epithelial defect - most frequently localized in the superior half cornea (Figure 2). Usually, around the epithelial defect, there is an area of poorly adherent opaque and oedematous epithelium that can spontaneously detach leading to an enlargement of the defect. Epithelial healing is inadequate and the edges of the defect become smooth and rolled. Descemet's membrane folds and stroma swelling may also be observed with an inflammatory reaction in the anterior chamber and, rarely, sterile hypopion may be observed.

Stage 3 is characterized by stromal involvement with a corneal ulcer that may progress to perforation and/or stromal melting (Figure 3). Corneal melting and perforation can be caused by inappropriate use of topical steroids or secondary infections (Figures 4 and 5).

\section{Diagnosis}

The diagnosis is frequently suspected on the basis of the patient's history. Systemic diseases such as diabetes 
Table 2 Clinical staging of neurotrophic keratitis

Stage 1

Rose bengal staining of the palpebral conjunctiva

Decreased break-up time

Increased viscosity of tear mucus

Punctate corneal keratopathy

Dellen

Small facets of drying epithelium (Gaule spots)

Superficial vascularization

Stromal scarring

Epithelial hyperplasia and irregularity

Hyperplastic precorneal membrane

Stage 2

Epithelial deficit, usually in the superior half cornea

Surrounding rim of loose epithelium

Stromal oedema

Anterior chamber inflammatory reaction, rare

Edges of the defect become smooth and rolled with time

Stage 3

Corneal ulcer

Stromal melting

Perforation

Adapted from Groos Jr EB. Neurotrophic keratitis. In: Krachmer JH, Mannis MJ, Holland EJ (eds). Cornea: Fundamentals of Cornea and External Disease, chap. 105. Mosby: St Louis, MO, 1997.

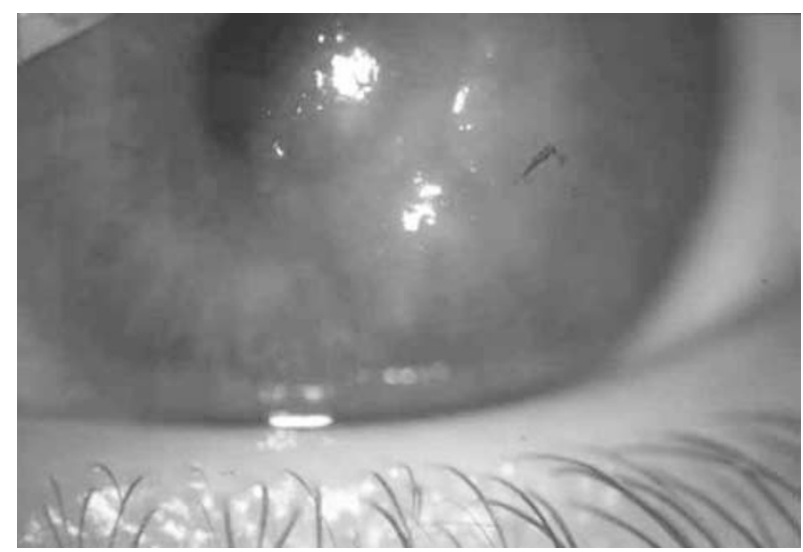

Figure 1 Hazy epithelium can be observed in stage 1 of neurotrophic keratitis.

mellitus, or systemic therapies (neuroleptic, antipsychotic, and antihistamine drugs) can cause impairment of trigeminal corneal innervation. Corneal surgery or traumas, topical anaesthetic abuse, chronic topical drugs, chemical burns, or contact lens abuse can cause local injury to corneal sensory nerves.

Brain neoplasms and vascular accidents may involve the fifth cranial nerve or its nucleus. Seventh and fifth cranial nerve lesions may occur during thyroid ophthalmopathy, periocular infiltrative neoplasm, or after surgical removal of acoustic neuroma. Aneurysms or cavernous sinus pathologies can cause ocular motility
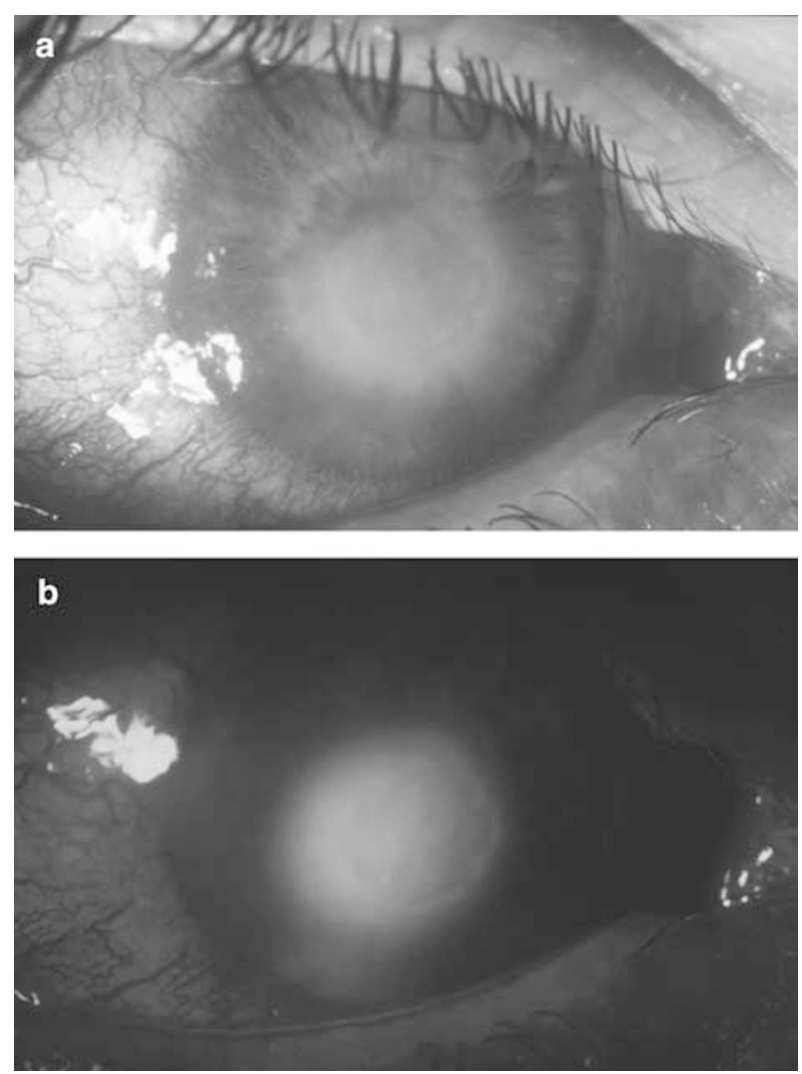

Figure 2 A large epithelial deficit in the central cornea is present in stage 2 of neurotrophic keratitis. Note the limits of the area of deficit with smooth, rolled, and loose epithelium. The epithelial defect (a) is highlighted by fluorescein staining (b).

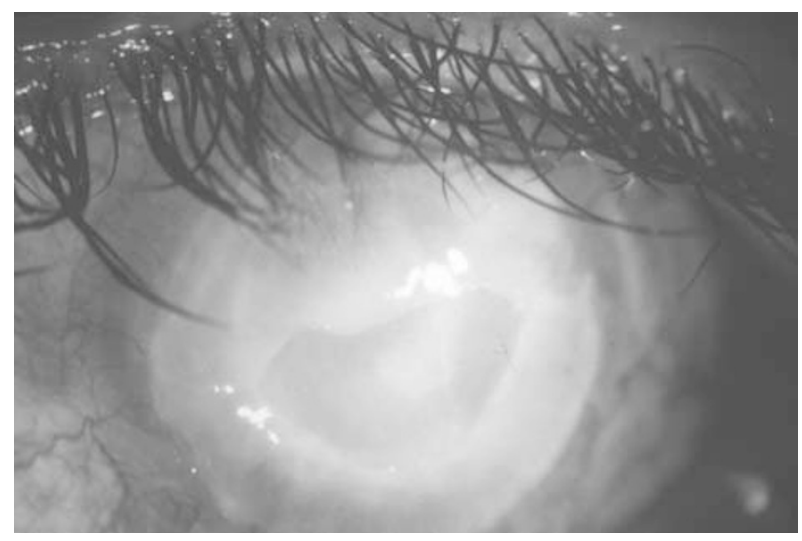

Figure 3 A circular ulcer is present in the central cornea in stage 3 of neurotrophic keratitis. The borders of the ulcer are clear-cut with no sign of epithelial healing.

alterations, while intraconal orbit lesions can be associated with optic nerve alterations and/or pupil abnormalities. The presence of neurological signs requires a strict collaboration with a neurologist and cranial imaging evaluation. 


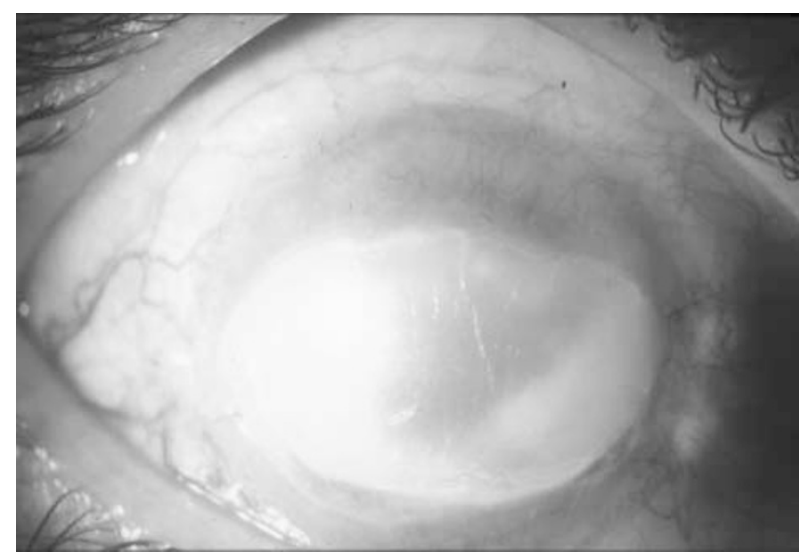

Figure 4 A severe complication of neurotrophic keratitis is the development of corneal stromal melting.

Accurate ocular examination must be carried out. Corneal sensitivity test may be performed by touching the central and the peripheral cornea with the tip of a cotton swab. ${ }^{26}$ Alternatively, the Cochet-Bonnet aesthesiometer may be used to localize and quantify the loss of corneal sensitivity, recording the patient's response at the touch of a nylon line (between 0 and $6 \mathrm{~cm}) .{ }^{27}$ Generally, the severity of neurotrophic keratitis is related to the severity of the corneal sensory impairment.

Blink rate is markedly decreased if bilateral neurotrophic keratitis occurs. However, the disease is frequently unilateral and blinks can be normal because the other unaffected eye elicits normal symmetrical blink.

A Schirmer test should be carried out because tear film production may be affected by the reduction of corneal sensitivity. ${ }^{28,29}$

Vital staining with fluorescein, rose bengal, or lissamine green shows corneal and conjunctival epithelial changes.

Accurate examination of the eyelids' margin, position, and motility is important because exposure keratitis and blepharitis can be associated with neurotrophic keratitis.

In all corneal ulcers, a microbiological examination should be performed to exclude bacterial, fungii, or viral infections.

\section{Differential diagnosis}

Punctate corneal keratopathy and tear film abnormalities, which characterize stage I of the disease, are also frequently observed in other ocular diseases such as dry eye, exposure keratitis, topical drug toxicity, contact lens abuse, and corneal limbal deficiency. The hallmark of neurotrophic keratitis is corneal anaesthesia; so the presence of ocular symptoms such as burning, foreign body sensation, photophobia, and dry eye orients the diagnosis towards other ocular surface diseases.
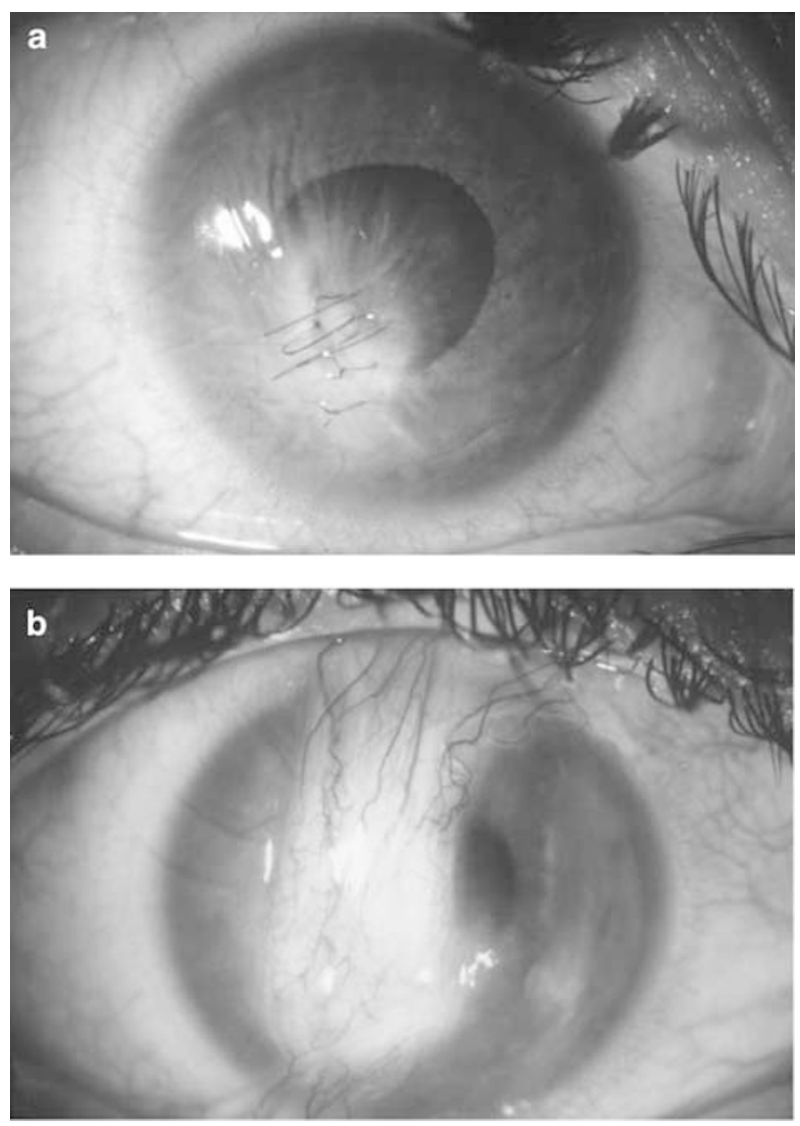

Figure 5 (a) Corneal perforation in a patient affected by neurotrophic keratitis treated with nylon sutures. This surgical treatment is inefficient in neurotrophic keratitis because of the impairment of the healing process. The same patient required a conjunctival flap to resolve the corneal perforation (b).

Clinical history, systemic signs and symptoms, and laboratory tests allow to address the correct diagnosis.

Superficial corneal vascularization and epithelial defects can also be seen in limbal stem cell deficiency. In this case, impression cytology may allow the identification of the epithelial phenotype by means of immunohistochemistry for cytokeratins (corneal epithelium is cytokeratin 3 positive and cytokeratin 19 negative, while conjunctival epithelium is cytokeratin 19 positive and cytokeratin 3 negative). ${ }^{30}$ However, diseases that cause limbal stem cell deficiency can damage sensory corneal nerves thus also causing neurotrophic keratitis.

Infective, toxic, or immune corneal ulcers always present important ocular inflammation, stromal infiltrates, and symptoms. Microbiologic examinations for bacteria, fungi, and viruses are always required. Any local treatment should be discontinued to exclude toxic corneal ulcers, and systemic evaluation for immune disorders should also be considered. 


\section{Treatment}

The goals of treatment of neurotrophic keratitis are to prevent progression of corneal damage and to promote epithelial healing. The therapy must be prompt and based on the clinical stage of the disease. ${ }^{31}$

The presence of an epithelial dystrophy or punctate keratopathy (stage 1) requires the discontinuation of all topical medications and the evaluation of side effects of systemic therapies such as neuroleptic, antipsychotic, and antihistamine drugs. Administration of topical preservative-free artificial tears may help in improving the corneal surface. The therapy at this stage aims to improve epithelial quality and transparency, and to avoid epithelial breakdown. Other ocular surface diseases such as exposure keratitis, dry eye, and limbal deficiency, if associated with neurotrophic keratitis, worsen the prognosis of the disease and require specific treatment. In these cases, it is important to correct the eyelid dysfunction, to consider punctal occlusion, and to consider limbal cell transplantation.

When an epithelial defect develops (stage 2), the aims of treatment are to avoid the development of corneal ulcer, to promote healing of the epithelial defect, and to prevent the recurrence of the epithelial breakdown. Withdrawal of all topical drugs is always required and preservative-free artificial tears can improve corneal surface. The patients should be carefully monitored because the progression of the disease is often without symptoms.

Corneal or scleral therapeutic contact lenses have been proposed, but their use may increase the risk of secondary infections and may cause sterile hypopyon. ${ }^{32-34}$

In the case of corneal ulcers that are unresponsive to artificial tears or therapeutic contact lenses, tarsorraphy is the most simple and diffuse procedure. If healing occurs, the tarsorraphy opening may be enlarged after a few weeks, but opening the tarsorraphy prematurely may result in a recurrence of corneal epithelial breakdown. Alternatively, it is possible to cover the epithelial defect by means of an amniotic membrane transplantation $^{35}$ or to utilize a palpebral spring or botulinum A toxin injection of the eyelid elevator. ${ }^{36,37}$

Topical steroids have been suggested because prostaglandins inhibit the epithelial growth and the use of steroids could reduce the activity of these inflammatory mediators, particularly in patients with chemical burns. ${ }^{13,38}$ However, steroids, by inhibiting stromal healing, may increase the risk of corneal stromal melting and perforation, thus their use should be considered with caution. Topical nonsteroid anti-inflammatory drug treatment does not induce improvement of the healing process. ${ }^{39}$
When a corneal ulcer develops (stage 3), the therapy is aimed at promoting corneal healing, and preventing corneal melting and perforation. As in stages 1 and 2, it is important to avoid the toxic effect of the topical treatment - thus only prophylactic topical antibiotic and preservative-free artificial tears are suggested. In the case of stromal melting, topical collagenase inhibitors, such as $\mathrm{N}$-acetylcysteine, tetracycline, or medroxyprogesterone may be administered. ${ }^{40}$ Tarsorraphy and conjunctival flap are effective surgical procedures in promoting corneal healing, but they give poor cosmetic outcome and the visual function is sacrificed. ${ }^{41,42}$ Small perforations can be treated with the application of cyanoacrylate glue followed by a soft bandage contact lens. ${ }^{43-45}$ Larger defects require lamellar or penetrating keratoplasty. ${ }^{44}$ The success rates of these corneal transplants are low because of poor wound healing and the persistent risk of epithelial defects owing to corneal anaesthesia (Figure 6). Reed et $a^{46}$ showed that $75 \%$ of 12 patients affected by corneal scarring secondary to herpes zoster keratitis and corneal hypoaesthesia maintained a clear corneal graft. The authors suggest that partial tarsorraphy may improve the success rate. However, the authors do not report the severity of corneal sensory impairment, which is, on the contrary, crucial for the long-term survival of penetrating keratoplasties.

\section{Prognosis}

Neurotrophic keratitis represents one of the most difficult and challenging ocular diseases still lacking treatment. The prognosis of neurotrophic keratitis depends on several factors such as the cause of the impairment of corneal sensitivity, the degree of corneal hypo/anaesthesia, and the association with other ocular

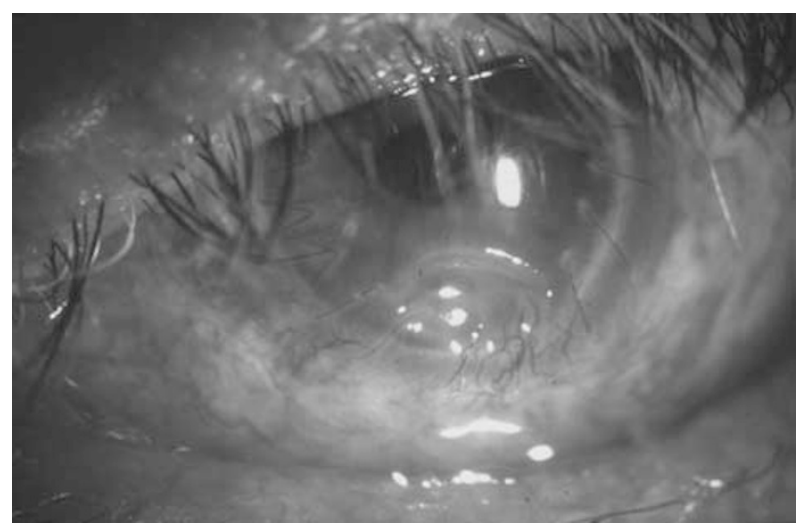

Figure 6 Lamellar and penetrating keratoplasties are high-risk surgical procedures in neurotrophic keratitis because of the frequent development of corneal epithelial defects, ulcers, and melting in the follow-up. The figure shows a relapse of neurotrophic corneal ulcer following penetrating keratoplasty. 
surface diseases such as dry eye, exposure keratitis, and limbal deficiency. It is generally accepted that the more severe the corneal sensory impairment, the higher the probability of disease progression. ${ }^{47}$

It is important to remind the patient to have himself checked frequently because the disease often lacks signs and symptoms, and protection with preservative-free artificial tears which may prevent epithelial breakdown is crucial. At present, the treatment of neurotrophic keratitis is still conservative and any surgical procedure aimed at restoring corneal transparency should be avoided because of the high risk of epithelial defect, ulcers, corneal melting, and perforation after surgery.

\section{Future developments and conclusions}

Several experimental and clinical studies have focused on new medical and surgical treatments for neurotrophic keratitis.

Experimental sympathectomy in the rabbit may reduce corneal changes after sensory nerve damage. ${ }^{18}$ However, surgical cervical sympathectomy did not find a practical application in humans and these findings are not confirmed by sympathectomy induced by chemical substances. ${ }^{48,49}$

Diabetic patients treated with topical aldose reductase inhibitor showed improvement of corneal sensitivity and morphologic characteristics of corneal epithelial cells. ${ }^{50}$

Several studies have suggested the use of neuropeptides and growth factors for the treatment of neurotrophic keratitis. Topical treatment with SP and IGF-1 induced corneal healing in two patients with neurotrophic keratitis. ${ }^{51}$ The authors reported no relapse of the disease within 1 year of follow-up. ${ }^{17,51}$

Epidermal growth factor has been used in human clinical trials with conflicting results. ${ }^{52,53}$ In an open uncontrolled study, topical nerve growth factor NGF treatment induced corneal healing in 43 patients affected by moderate and severe neurotrophic keratitis (stages 2 and 3) ${ }^{19,20}$ Restoration of corneal integrity was associated with recovery of corneal sensitivity in most of the patients. Topical treatment with NGF represents a promising therapy to prevent the onset and progression of neurotrophic keratitis. NGF modulates the function, regeneration, and survival of sensory nerves; it supports epithelial proliferation and differentiation, and it influences the production and release of neuropeptides.

In conclusion, the management of neurotrophic keratitis represents a real therapeutic dilemma for ophthalmologists. Several medical and surgical treatments have been proposed to halt the progression of the disease and to avoid corneal perforation. These surgical procedures can preserve ocular integrity but they often sacrifice cosmetic appearance and visual function. Neuropeptides and growth factors may represent a future therapeutic approach for the cure and prevention of neurotrophic keratitis.

\section{References}

1 Groos Jr EB. Neurotrophic keratitis. In: Krachmer JH, Mannis MJ, Holland EJ (eds.) Cornea: Clinical Diagnosis and Management. Mosby: St Louis, 1997, pp 1340.

2 Puca A, Meglio M, Vari R, Tamburrini G, Tancredi A. Evaluation of fifth nerve dysfunction in 136 patients with middle and posterior cranial fossae. Eur Neurol 1995; 35: 33-37.

3 Hyndiuk RA, Kazarian EL, Schultz RO, Seideman S. Neurotrophic corneal ulcers in diabetes mellitus. Arch Ophthalmol 1977; 95: 2193-2196.

4 Kara-corlu MA, Cakiner T, Saylan T. Neurotrophic corneal ulcers in diabetes mellitus. Arch Ophthalmol 1977; 95: 2193-2196.

5 Mackie IA. Neuroparalytic keratitis. In: Fraunfelder F, Roy FH, Meyer SM (eds). Current Ocular Therapy. WB Saunders: Philadelphia, PA, 1995, pp 452-454.

6 Sigelman S, Friedenwald JS. Mitotic and wound healing activities of the corneal epithelium: effect of sensory denervation. Arch Ophthalmol 1954; 52: 46-57.

7 Simone S. De ricerche sul contenuto in acqua totale ed in azoto totale della cornea di coniglio in condizione di cheratite neuroparalitica sperimentale. Arch Ottalmol 1958; 62: 151.

8 Alper MG. The anhestetic eye: an investigation of changes in the anterior ocular segment of the monkey caused by interrupting the trigeminal nerve at various levels along its course. Trans Am Ophthalmol Soc 1975; 73: 323-365.

9 Mackie IA. Role of the corneal nerves in destructive disease of the cornea. Trans Ophthalmol Soc UK 1978; 93: 343.

10 Gilbard JP, Rossi SR. Tear film and ocular surface changes in a rabbit model of neurotrophic keratitis. Ophthalmology 1990; 97: 308-312.

11 Mishima S. The effects of the denervation and the stimulation of the sympathetic and trigeminal nerve on the mitotic rate of the corneal epithelium in the rabbit. Jpn J Ophthalmol 1957; 1: 65-73.

12 Shimizu T, Izumi K, Fuijta S, Koja T, Sorimachi M, Ohba N et al. capsaicin-induced corneal lesions in mice and the effects of chemical sympathectomy.J Pharmacol Exp Ther 1987; 243: 690-695

13 Cavanagh HD, Colley AM. The molecular basis of neurotrophic keratitis. Acta Ophthalmol Suppl 1989; 192: 115-134.

14 Mikulec AA, Tanelian DL. CGRP increases the rate of corneal re-epithelization in an in vitro whole mount preparation. J Ocul Pharmacol Ter 1996; 12: 417-423.

15 Reid TW, Murphy CJ, Iwahashi CK, Foster BA, Mannis MJ. Stimulation of epithelial cell growth by the neuropeptide substance P. J Cell Biochem 1993; 52: 476-485.

16 Kingsley RE, Marffurt CF. Topical substance P and corneal epithelia wound closure in the rabbit. Invest Ophthalmol Vis Sci 1997; 38: 388-395.

17 Chikama T, Fukuda K, Morishige N, Nishida T. Treatment of neurotrophic keratopathy with substance-P-derived peptide (FGLM) and insulin growth factor 1. Lancet 1998; 351: $1783-1784$ 
18 Fujita S, Miyazono Y, Ohba N. Capsaicin-induced corneal changes associated with sensory denervation in neonatal rat. Jpn J Ophthalmol 1987; 31: 412-424.

19 Bonini S, Lambiase A, Rama P, Caprioglio G, Aloe L. Topical treatment with nerve growth factor for neurotrophic keratitis. Ophthalmology 2000; 107, 1347-1351, discussion 1351-1352.

20 Lambiase A, Rama P, Bonini S, Caprioglio G, Aloe L. Topical treatment with nerve growth factor for corneal neurotrophic ulcers. N Engl J Med 1998; 338: 1174-1180.

21 Donnerer J, Amann R, Schuligoi R, Skofitsch G. Complete recovery by nerve growth factor of neuropeptide content and function in capsaicin-impaired sensory neurons. Brain Res 1996; 741: 103-108.

22 Levi-Montalcini R. The nerve growth factor 35 years later. Science 1987; 237: 1154-1162.

23 Schicho R, Skofitsch G, Donnerer J. Regenerative effect of human recombinant NGF on capsaicin-lesioned sensory neurons in the adult rat. Brain Res 1999; 815: 60-69.

24 de Castro F, Silos-Santiago I, Lopez de Armentia M, Barbacid M, Belmonte C. Corneal innervation and sensitivity to noxious stimuli in trkA knockout mice. Eur J Neurosci 1998; 10: 146-152.

25 Kruse FE, Tseng SCG. Growth factors modulate clonal growth and differentiation of cultured rabbit limbal and corneal epithelium. Invest Ophthalmol Vis Sci 1993; 34: 1963-1976.

26 Faulkner WJ, Varley GA. Corneal diagnostic tecnique. In: Krachmer JH, Mannis MJ, Holland EJ (eds). Cornea: Foundamentals of Cornea and External Disease. Mosby: St Louis, MO, 1997, pp 275-281.

27 Norn MS. Measurement of sensitivity. In: Norn MS (ed). External Eye Diseases. Methods of Examination. Munksgaard International Publisher Ltd: Copenhagen, 1974

28 Heigle TJ, Pflugfelder SC. Aqueous tear production in patients with neurotrophic keratitis. Cornea 1996; 15: 135-138.

29 Wright P, Mackie IA. Mucus in the healthy and diseased eye. Trans Ophthalmol Soc UK 1977; 97: 1-7.

30 Elder MJ, Hiscott P, Dart JK. Intermediate filament expression by normal and diseased human corneal epithelium. Hum Pathol 1997; 28: 1348-1354.

31 Lambiase A, Rama P, Aloe L, Bonini S. Management of neurotrophic keratopathy. Curr Opin Ophthalmol 1999; 10: $270-276$.

32 Gould HL. Treatment of neurotrophic keratitis with scleral contact lenses. Eye Ear Nose Throat Mon 1967; 46: 1406-1414.

33 Kent HD, Cohen EJ, Laibson PR, Arentsen JJ. Microbial keratitis and corneal ulceration associated with therapeutic soft contact lenses. CLAO J 1990; 16: 49-52.

34 Pfister RR. Clinical measures to promote corneal epithelial healing. Acta Ophthalmol Suppl 1992; 202: 73-83.

35 Lee SH, Tseng SC. Amniotic membrane transplantation for persistent epithelial defects with ulceration. Am J Ophthalmol 1997; 123: 303-312.
36 Kirkness CM, Adams GGW, Dilly PN, Lee JP. Botulinum toxin A-induced protective ptosis in corneal disease. Ophthalmology 1988; 95: 473-480.

37 Mc Neill JI, Oh YH. An improved palpebral spring for the management of paralytic lagophthalmos. Ophthalmology 1991; 98: 715-719.

38 Cavanagh HD, Colley AM, Pihlaja DJ. Persistent corneal epithelial defects. Int Ophthalmol Clin 1979; 19: 197-206.

39 Hersh PS, Rice BA, Baer JC, Wells PA, Lynch SE, McGuigan LJB et al. Topical nonsteroidal agents and corneal wound healing. Arch Ophthalmol 1990; 108: 577-583.

40 Davis EA, Dohlman CH. Neurotrophic keratitis. Int Ophthalmol Clin 2001; 41: 1-11.

41 Gundersen T, Pearlson HR. Conjunctival flaps for corneal diseases: their usefulness and complications. Trans Am Ophthalmol Soc 1969; 67: 78-95.

42 Lugo M, Arentsen JJ. Treatment of neurotrophic ulcers with conjunctival flaps. Am J Ophthalmol 1987; 103: 711-712.

43 Fogle JA, Kenyon KR, Foster CS. Tissue adhesive arrests stromal melting in the human cornea. Am J Ophthalmol 1980; 89: 795-802.

44 Hirst LW, Smiddy WE, Stark WJ. Corneal perforations: changing methods of treatment, 1960-1980. Ophthalmology 1982; 89: 630-634.

45 Webster RG, Slansky HH, Refojo MF, Boruchoff SA, Dohlman $\mathrm{CH}$. The use of adhesive for the closure of corneal perforation. Arch Ophthalmol 1968; 80: 705-709.

46 Reed JW, Joyner SJ, Knauer III WJ. Penetrating keratoplasty for herpes zoster keratopathy. Am J Ophthalmol 1989; 107: 257-261.

47 Cobo LM. Corneal complications of herpes zoster ophthalmicus. Prevention and treatment. Cornea 1988; 7: $50-56$.

48 Adams GG, Cullen JF. Neuroparalytic keratitis and the effect of cervical sympathectomy following operative procedurs for trigeminal neuralgia. Scott Med J 1987; 32: $86-88$.

49 Welch E, Geary J. Current status of thoracic dorsal sympathectomy. J Vasc Surg 1984; 1: 202-214.

50 Hosotani H, Ohashi Y, Yamada M, Tsubota K. Reversal of abnormal corneal epithelial cell morphologic characteristics and reduced corneal sensitivity in diabetic patients by aldose reductase inhibitor, CT-112. Am J Ophthalmol 1995; 119: 288-294

51 Brown SM, Lamberts DW, Reid TW, Nishida T, Murphy CJ. Neurotrophic and anhydrotic keratopathy treated with substance P and insulin like growth factor 1. Arch Ophthalmol 1997; 115: 926-927.

52 Daniele S, Gilbard JP, Schepens CL. Treatment of persistent epithelial defects in neurotrophic keratitis with epidermal growth factor: a preliminary open study. Graefes Arch Clin Exp Ophthalmol 1992; 230: 314-317.

53 Kandarakis AS, Page C, Kaufman HE. The effect of epidermal growth factor on epithelial healing after penetrating keratoplasty in human eyes. Am J Ophthalmol 1984; 98: 411-415. 